\title{
DEGRADACIÓN ACELERADA DE PELÍCULAS DE POLIETILENO CON QUITOSANO COMPATIBILIZADAS CON ANHÍDRIDO MALÉICO
}

\author{
Dora Evelia RODRÍGUEZ FÉLIX ${ }^{1}$, Jesús Manuel QUIROZ CASTILLO ${ }^{2 *}$, \\ María Monica CASTILLO ORTEGA ${ }^{1}$, Lauren Lucero LIZÁRRAGA LABORÍN ${ }^{1}$, \\ Thania GARCÍA DUARTE², Daniel GARCÍA BEDOYA², Martín Eusebio CRUZ CAMPAS ${ }^{2}$, \\ Roberto RAMÍREZ LEAL ${ }^{2}$ y Pedro Jesús HERRERA FRANCO ${ }^{3}$
}

${ }^{1}$ Universidad de Sonora. Rosales y Luis Encinas, Colonia Centro, Hermosillo, Sonora, México, C.P. 83000

${ }^{2}$ Universidad Estatal de Sonora. Ley Federal del Trabajo e Israel González, Colonia Apolo, Hermosillo, Sonora, México, C.P. 83100

${ }^{3}$ Centro de Investigación Científica de Yucatán. Calle 43 No. 130, Colonia Chuburná de Hidalgo, Mérida, Yucatán, C.P. 97200

*Autor para correspondencia: quiroz51@hotmail.com

(Recibido julio 2015; aceptado noviembre 2015)

Palabras clave: extrusión, propiedades mecánicas, espectroscopía de infrarrojo, degradación en tierra

\section{RESUMEN}

La utilización de polímeros sintéticos como materia prima para empaques desechables genera altos volúmenes de contaminantes persistentes en el ambiente. Una manera de resolver el problema del acumulamiento de plásticos, es la producción de materiales poliméricos biodegradables. En este trabajo se presenta la preparación y pruebas de degradación acelerada de películas preparadas a partir de mezclas de polietileno lineal de baja densidad con quitosano, utilizando anhídrido maléico como compatibilizante. Las películas de polietileno con un $15 \%$ de quitosano perdieron $40 \%$ del valor inicial de resistencia a la tensión después de $360 \mathrm{~h}$ de degradación acelerada. En contraste con el $20 \%$ de pérdida que presentó la película de polietileno puro durante el mismo periodo. Las películas con quitosano, además, redujeron $13 \%$ de su peso después de estar bajo tierra de vivero durante 180 días, un valor considerablemente mayor que el $0.05 \%$ que presentó la película de polietileno. La presencia de anhídrido maléico como compatibilizante favoreció un ligero aumento en la degradación de las películas de polietileno con quitosano.

Key words: extrusion, mechanical analysis, infrared spectroscopy, degradation in soil

\begin{abstract}
The use of synthetic polymers as material for the production of disposable packaging, generates high volumes of persistent environmental pollutants. One way to solve the problem of the accumulation of plastics is the production of biodegradable polymeric materials. This work presents the preparation and testing of the degradation capability of films prepared from blends of linear low density polyethylene with chitosan, using maleic anhydride as a compatibilizer. Polyethylene films with $15 \%$ chitosan, lost $40 \%$ of the initial values of tensile strength after $360 \mathrm{~h}$ of accelerated degradation, in contrast
\end{abstract}


to the $20 \%$ loss by the pure polyethylene film. Chitosan films also showed a $13 \%$ of weight loss after being buried for 180 days, significantly more than the $0.05 \%$ registered for the polyethylene film. The presence of maleic anhydride as a compatibilizing agent had a slight effect on the degradation rate of polyethylene films with chitosan.

\section{INTRODUCCIÓN}

Al utilizar polímeros sintéticos, surge como un problema asociado, la contaminación ambiental como producto del desecho de los plásticos. Estos polímeros son resistentes a la biodegradación debido principalmente a que son hidrofóbicos y tienen altos pesos moleculares. Una gran cantidad de los plásticos producidos son destinados a utilizarse como empaque desechable, los cuales permanecen en el ambiente después de su uso. El desecho de estos plásticos trae consecuencias negativas a las comunidades humanas debido la acumulación de basura y estancamiento de aguas negras o pluviales al cerrar o bloquear su paso con dicha acumulación.

Uno de los polímeros sintéticos más utilizados a nivel mundial, es el polietileno (Dostál et al. 2008), que presenta una buena resistencia química, es inodoro y no es tóxico. Sin embargo, es conocido por su alta resistencia a la degradación (Singh et al. 2011).

La necesidad de resolver este problema de contaminación ha estimulado el desarrollo de materiales poliméricos biodegradables. Los residuos de estos materiales son capaces de sufrir degradación cuando están expuestos a factores naturales (rayos ultravioleta y microorganismos presentes en el suelo y en el aire), así como a reactivos químicos solubles en agua. Una gran variedad de polímeros sintéticos y naturales absorben radiación ultravioleta (UV) y sufren reacciones fotolíticas, termo y foto-oxidativas (Gugumus 1993, Andrady 1997, Andrady et al. 1998). Un importante grupo de materiales poliméricos biodegradables resultan de las mezclas basadas en termoplásticos sintéticos y polisacáridos naturales, como celulosa, almidón, quitina, quitosano, entre otros (Ermolovich y Makarevich 2006).

La matriz polimérica utilizada para la preparación de películas en este trabajo fue el polietileno lineal de baja densidad (PE). Este polímero cuenta con un esqueleto lineal con ramificaciones laterales muy cortas y uniformes, que hacen que su temperatura de fusión y su resistencia a la tensión sean superiores a la del polietileno de baja densidad convencional (Guadagno et al. 2001, Corrales et al. 2002). El polímero biodegradable que se seleccionó para ser agregado a la matriz polimérica de PE fue el quitosano. Este polímero es uno de los polisacáridos que se encuentra con mayor frecuencia en la naturaleza y tiene un gran potencial para utilizarse como material de empaque debido a su capacidad de biodegradarse. Además, es biocompatible y cuenta con actividad antimicrobiana (Harish-Prashant y Tharanathan 2007, Nair y Laurencin 2007, Mathew y Abrahan 2008, Pillai et al. 2009, Zhong et al. 2010).

El objetivo de este trabajo fue evaluar la capacidad de degradación de las mezclas de PE/quitosano en una cámara de degradación acelerada, así como evaluar el efecto de la adición del polietileno injertado con anhídrido maléico (PEgMA) como compatibilizante sobre las propiedades físicas, químicas y mecánicas de las mezclas resultantes.

\section{MATERIALES Y MÉTODOS}

Los siguientes materiales fueron obtenidos de la compañía Sigma-Aldrich: quitosano (peso molecular promedio de $250 \pm 60 \mathrm{kDa}$ y una desacetilación de $80 \pm 5 \%$ ), polietileno injertado con anhídrido maléico (PEgMA, con un 3.5\% de anhídrido maléico) y glicerol.

El PE grado comercial (con un índice de flujo fundido de $2.0 \mathrm{~g} / 10$ min con un dado estándar de $2.16 \mathrm{~kg}$ a $190{ }^{\circ} \mathrm{C}$ ) se obtuvo de la Compañía Petroquímica Qatar (QAPCO, por sus siglas en inglés). El quitosano fue secado en una estufa a $50{ }^{\circ} \mathrm{C}$ por $24 \mathrm{~h}$ antes de usarse para reducir la presencia de humedad y el PE fue molido previamente.

\section{Preparación de películas}

Las mezclas de PE y quitosano fueron preparadas con glicerol como plastificante. Se seleccionó el glicerol debido a que su capacidad como plastificante ha sido documentada en la literatura, además de su inocuidad y su buena estabilidad térmica (Ermolovich y Makarevich 2006).

Se prepararon películas de PE y quitosano sin compatibilizante y películas en las que se agregó PEgMA como un compatibilizante para la mezcla. Posteriormente, se comparó su comportamiento mecánico. Las mezclas poliméricas fueron preparadas 
siguiendo una modificación del procedimiento reportado por Quiroz-Castillo et al. (2014), que fue en dos etapas: (1) se mezcló el quitosano con el plastificante hasta obtener una masa, la modificación del procedimiento reportado consistió en la inclusión de una etapa de agitación mecánica por $20 \mathrm{~min}$, para asegurar la homogeneidad de dicha mezcla y (2) se mezcló el quitosano y glicerol con el PE. En las mezclas compatibilizadas, el PEgMA fue adicionado en la primera etapa. Para ambos casos, las mezclas fueron agitadas mecánicamente por 30 min y después extrudidas con un mezclador-extrusor de laboratorio marca Atlas, a una velocidad de rotación de $40 \mathrm{rpm}$. Las temperaturas fueron controladas a 130 y $140{ }^{\circ} \mathrm{C}$ para el rotor y el cabezal, respectivamente, excepto para la película de PE puro, que en este caso, las temperaturas fueron controladas a 115 y $125^{\circ} \mathrm{C}$. En el cuadro I se indican las composiciones para cada mezcla preparada. La relación glicerol/quitosano se mantuvo constante en todas las mezclas, siendo de $2 \mathrm{~g}$ de glicerol/1g de quitosano.

CUADRO I. IDENTIFICACIÓN DE PELÍCULAS DE POLIETILENO Y QUITOSANO

\begin{tabular}{|c|c|c|c|c|}
\hline \multirow{2}{*}{ Código } & \multicolumn{4}{|c|}{$\begin{array}{l}\text { Composición de películas de polietileno, } \\
\text { quitosano y polietileno injertado con anhídrido } \\
\text { maléico (PegMA) }\end{array}$} \\
\hline & $\begin{array}{c}\text { Polietileno } \\
(\%)\end{array}$ & $\begin{array}{l}\text { Quitosano } \\
(\%)\end{array}$ & $\begin{array}{c}\text { PEgMA } \\
(\%)\end{array}$ & $\begin{array}{c}\text { Glicerol }(\mathrm{g} / \mathrm{g} \\
\text { quitosano) }\end{array}$ \\
\hline $\mathrm{PE}$ & 100 & 0 & 0 & 0 \\
\hline A & 85 & 15 & 0 & 2 \\
\hline B & 80 & 15 & 5 & 2 \\
\hline
\end{tabular}

\section{Caracterización}

\section{Estudios de degradación acelerada}

Las películas se cortaron en probetas (geometría y dimensiones de acuerdo con la norma ASTM D1708), estas películas fueron expuestas en una cámara de degradación acelerada QUV, marca Q-LAB, modelo QUV/SE, donde se produjo radiación UV a través de 8 lámparas UVA-340, con una potencia total de $3.56 \mathrm{~W} / \mathrm{m}^{2}$, a una longitud de onda de 365 a $295 \mathrm{~nm}$. Durante el experimento, se mantuvo una temperatura constante de $60^{\circ} \mathrm{C}$ dentro de la cámara. El programa de degradación acelerada consistió en ciclos de $8 \mathrm{~h}$ con radiación UV seguidos de $8 \mathrm{~h}$ sin radiación UV, donde se agregó un rocío de agua para alcanzar una humedad relativa de $100 \%$, para después repetir el ciclo. A las 180, 270 y 360 h, 10 especímenes de cada tipo de película fueron retirados de la cámara y sometidos a las siguientes técnicas de caracterización: espectroscopía de infrarrojo de transformada de Fourier, ensayos de resistencia a la tensión y observación visual (registrada en fotografías).

\section{Espectroscopía de infrarrojo}

La espectroscopía de infrarrojo de transformada de Fourier (FT-IR) de las películas se llevó a cabo en un espectrofotómetro FT-IR Perkin-Elmer 1600. Se

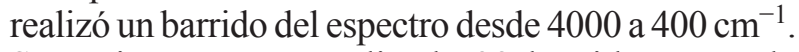
Se registró un promedio de 32 barridos. Para la espectroscopía se utilizó una pastilla de $\mathrm{KBr}$ con aproximadamente $5 \mathrm{mg}$ de muestra y se realizó una medición en modo de transmitancia (Del CastilloCastro et al. 2011).

\section{Propiedades mecánicas}

Las propiedades mecánicas de las películas fueron medidas en el modo de resistencia a la tensión, con una máquina universal United SSTM- $5 \mathrm{kN}$ a una velocidad de desplazamiento del cabezal de $10 \mathrm{~mm} /$ min. Se reportan los valores promedio de al menos ocho especímenes de cada película. El espesor de las películas fue medido con un micrómetro Mitutoyo con una precisión de $0.001 \mathrm{~mm}$ (Del Castillo-Castro et al. 2011).

\section{Observación visual}

Se seleccionó un espécimen representativo de cada composición de las películas preparadas para estudiar las características de su superficie a través de la observación visual registrada en fotografías capturadas con una cámara digital de 15 megapixeles.

\section{Estudios de degradación en tierra}

Se seleccionaron 10 probetas cualitativamente representativas de las películas, se lavaron con agua destilada y se secaron por $24 \mathrm{~h}$ a $50{ }^{\circ} \mathrm{C}$ en un horno de convección por gravedad marca Felisa, modelo FE0293.

Se obtuvo tierra con abono comercial de un vivero local, se pesaron $2 \mathrm{~kg}$ y se colocaron en una bolsa de polietileno de $30 \times 40 \mathrm{~cm}$. Posteriormente se agregaron $400 \mathrm{~g}$ de agua de la llave (aproximadamente $20 \%$ de humedad). Las probetas fueron colocadas en una almáciga donde se colocó la tierra y fueron retiradas cada 45 días hasta alcanzar los 180 días. En cada periodo, las películas se lavaron con agua destilada, se secaron por $24 \mathrm{~h}$ a $50{ }^{\circ} \mathrm{C}$ y después se pesaron en una balanza Sartorius modelo R200D con una precisión de $0.00001 \mathrm{~g}$. Después de pesarse, las probetas fueron colocadas nuevamente en la tierra (Chuensangjun et al. 2013) 


\section{RESULTADOS Y DISCUSIÓN}

\section{Estudios de degradación acelerada} Análisis de espectroscopía de infrarrojo

En la figura 1 se presentan los espectros de FT-IR de las películas antes y después de la degradación acelerada. El intervalo presentado de número de onda es de $4000-400 \mathrm{~cm}^{-1}$ (Fig. 1a, Fig. 2a y Fig. 3a) y de $2000-1500 \mathrm{~cm}^{-1}$ (Fig. 1b, Fig. 2b y Fig. 3b), esta amplificación del intervalo se presenta ya que es donde se pueden apreciar los cambios estructurales debidos a la fotodegradación.

La figura 1a banda 1, muestra los picos característicos del PE: (1) pico de estiramiento del hidrocarburo en un número de onda alrededor de $3000-2800 \mathrm{~cm}^{-1}$, (2) pico de movimiento de tijereteo del metileno a

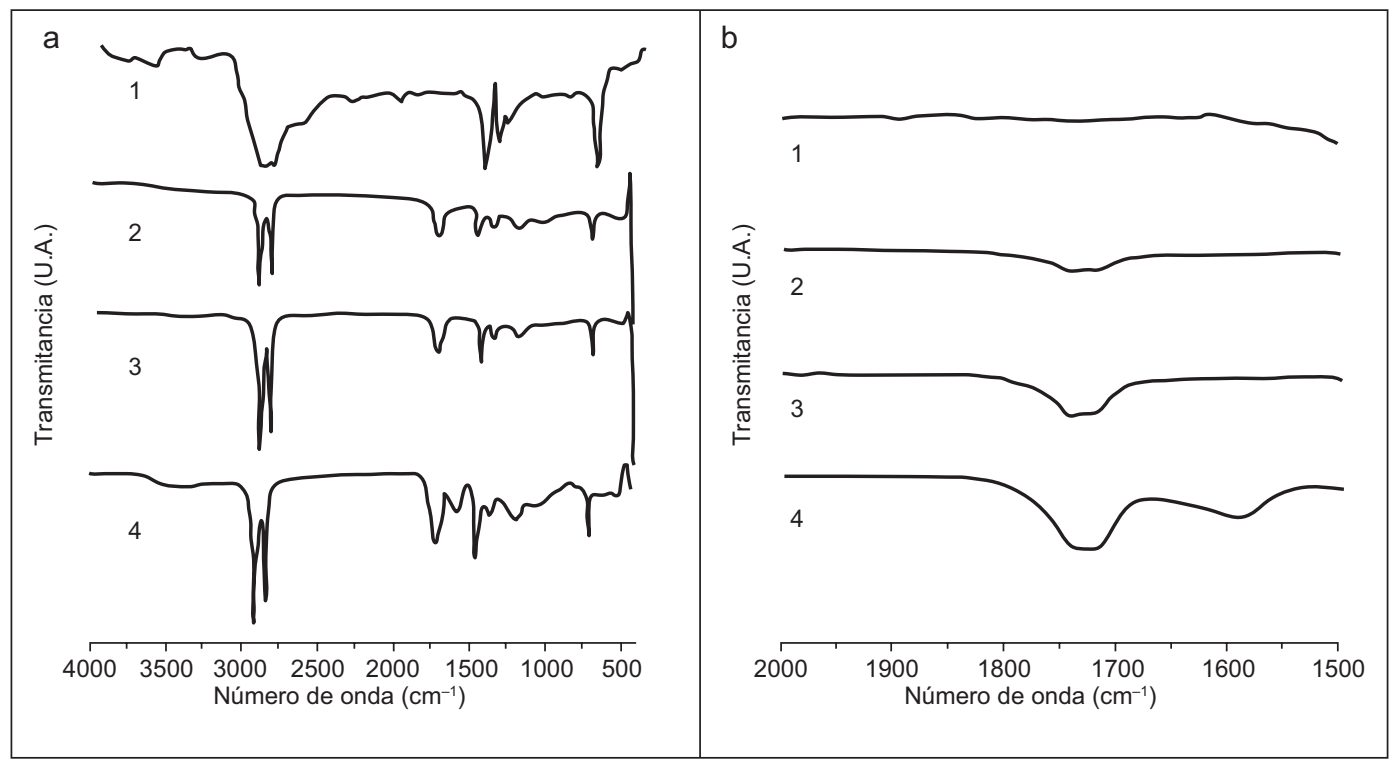

Fig. 1. Espectros de infrarrojo de transformada de Fourier (a) de 4000 a $400 \mathrm{~cm}^{-1}$ y (b) de 2000 a $1500 \mathrm{~cm}^{-1}$, de la película de polietileno registrados a $0,180,270$ y 360 h de degradación acelerada (1-4, respectivamente). U.A. $=$ uniddes arbitrarias

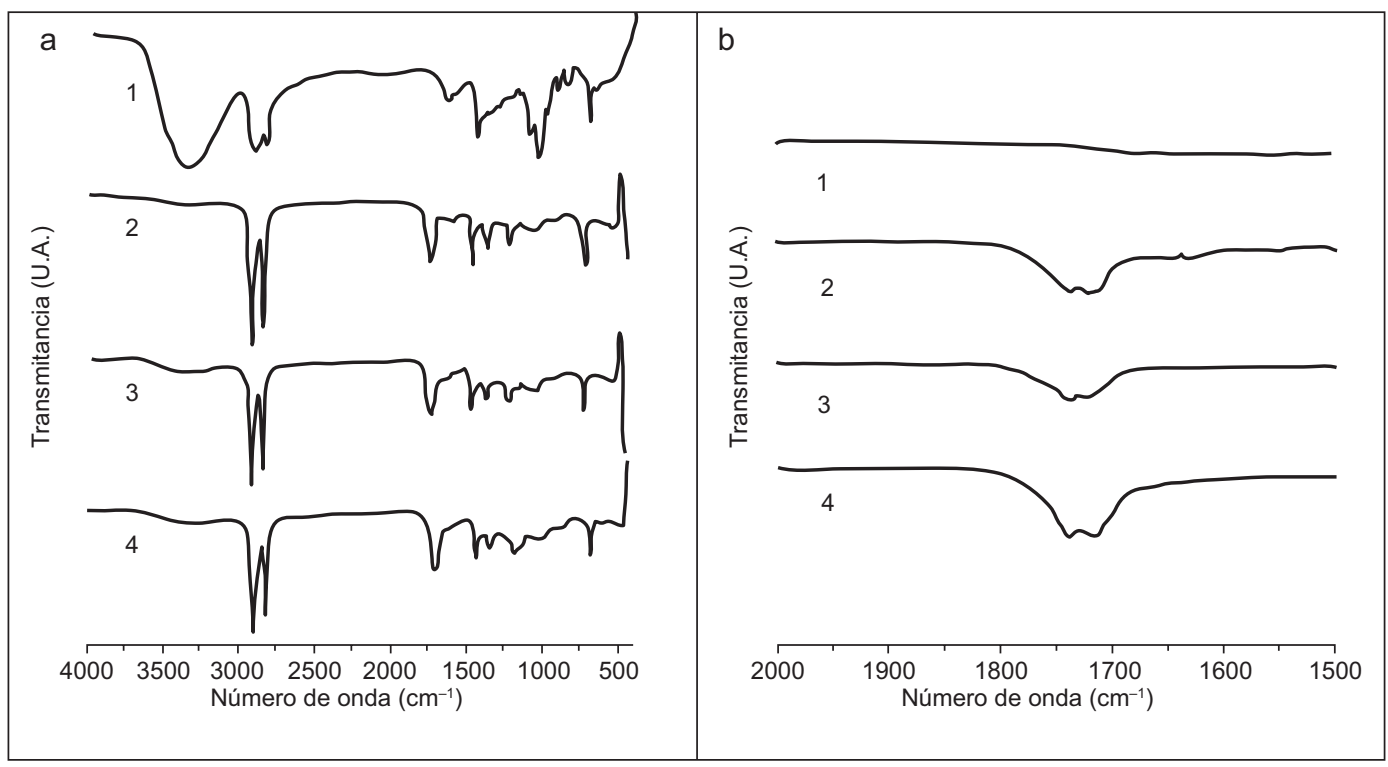

Fig. 2. Espectros de infrarrojo de transformada de Fourier (a) de 4000 a $400 \mathrm{~cm}^{-1}$ y (b) de 2000 a $1500 \mathrm{~cm}^{-1}$, de la película A, registrados a 0,180, 270 y 360 horas de degradación acelerada (1-4, respectivamente). U.A. $=$ uniddes arbitrarias 


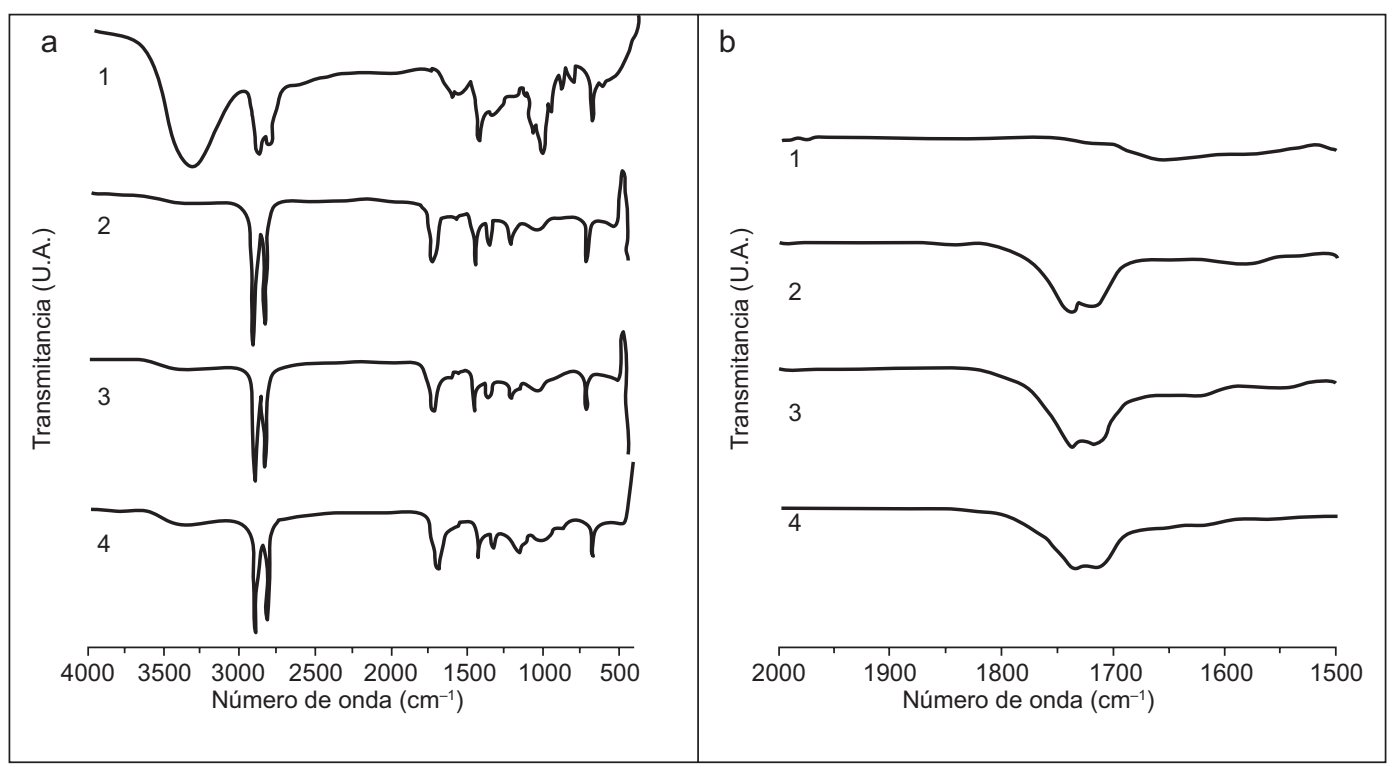

Fig. 3. Espectros de infrarrojo de transformada de Fourier (a) de 4000 a $400 \mathrm{~cm}^{-1}$ y (b) de 2000 a $1500 \mathrm{~cm}^{-1}$, de la película $\mathrm{B}$, registrados a $0,180,270$ y 360 horas de degradación acelerada (1-4, respectivamente). U.A. $=$ uniddes arbitrarias

$1467 \mathrm{~cm}^{-1}$ y (3) banda de balanceo del metileno a $722 \mathrm{~cm}^{-1}$ (Del Castillo-Castro et al. 2011). Después de 180 h de degradación acelerada de las películas de PE puro, apareció una nueva banda a $1712 \mathrm{~cm}^{-1}$ (Fig. 1a y b, banda 2), la cual indica la presencia de grupos carbonilo. La aparición de este grupo es un cambio estructural esperado por efecto de la fotodegradación. Cabe resaltar que el área relativa de esta banda aumentó conforme avanzó el tiempo de exposición. Estos resultados son consistentes con literatura científica relacionada con el mecanismo de degradación del PE (Day et al. 1997, Dintcheva et al. 2009, Ojeda et al. 2011, Dintcheva et al. 2010).

Antes de la degradación acelerada, los espectros de las películas con quitosano (Fig. 2a y b, 3a y b, banda 1) mostraron las bandas características de los polímeros individuales, como el estiramiento del grupo $\mathrm{OH}$ del quitosano a los $3365 \mathrm{~cm}^{-1}$ y las bandas a los 2932,2887 y $1635 \mathrm{~cm}^{-1}$ que representan la presencia de los grupos $-\mathrm{CH}_{2}-,-\mathrm{CH}_{3}$ y $-\mathrm{NH}_{2}$, respectivamente (Sionkowska et al. 2011). Después de $180 \mathrm{~h}$ de degradación acelerada, la presencia del grupo carbonilo es evidente en los espectros, la banda que indica este cambio estructural apareció a $\operatorname{los} 1712 \mathrm{~cm}^{-1}$ en ambas películas, compatibilizadas (B) (Fig. 3a y b, banda 2)y no compatibilizadas (A) (Fig. 2a yb, banda 2). Después de $360 \mathrm{~h}$ de degradación acelerada se puede observar un aumento en el área de esta señal (Fig. 2a y b, banda 4 y Fig. 3a y b, banda 4). Esta área siguió aumentando en relación con el tiempo de degradación, lo que indica un incremento en los grupos carbonilo presentes en la película debido a la fotodegradación de la muestra. El área de la banda siguió aumentando en relación con el tiempo de degradación.

Las películas con quitosano (Fig. 2 y Fig. 3) sufrieron una rápida oxidación, evidenciada por el aumento del área de la banda del grupo carbonilo. Los productos de la fotooxidación del PE aparecen también en las películas que contienen quitosano (Fig. 2a y b, banda 4), independientemente de la presencia de PEgMA (Fig. 3a y b, banda 4).

Aparentemente el compatibilizante no tuvo un papel determinante en el mecanismo de degradación. Sin embargo, favoreció ligeramente la oxidación de los polímeros con quitosano. Este efecto del PEgMA en la velocidad de degradación puede deberse a la inestabilidad fotooxidativa del anhídrido maléico y a las posibles interacciones entre el grupo anhídrido y el quitosano (Rogovinaa et al. 2009). La baja interacción entre las fases de las películas sin compatibilizante (A), podría producir un aumento en la resistencia a la degradación.

La deshidratación y la despolimerización son consideradas generalmente como los dos procesos principales en el mecanismo de degradación de los polisacáridos (Freile-Pelegrin et al. 2007). Con la adición de quitosano, la transmitancia de los grupos carbonilo en los espectros de las películas sufre alteraciones similares que las observadas para la película de PE puro (Fig. 3a y b, bandas 2-4). 


\section{Análisis de propiedades mecánicas}

La figura 4 muestra los valores adimensionales de retención para el módulo de Young (MY), resistencia a la tensión (RT) y deformación a la ruptura (DR), reportados en función del tiempo de degradación acelerada. Esta retención de propiedades fue calculada dividiendo los valores de MY, RT y DR a los diferentes tiempos de degradación entre los valores que presentó el material antes de la degradación acelerada. Las propiedades mecánicas, son usadas comúnmente para monitorear el comportamiento de degradación de un material debido a su alta sensibilidad a las variaciones estructurales y morfológicas que ocurren durante la fotooxidación (Day et al. 1997).

Las propiedades mecánicas de las películas cambiaron al aumentar el tiempo de degradación en la cámara QUV (Fig. 4). Para las películas de PE puro, el MY disminuyó $22 \%$ después de $180 \mathrm{~h}$ de exposición, este valor se mantuvo constante a las $270 \mathrm{~h}$ para después disminuir $38 \%$ al final del experimento después de $360 \mathrm{~h}$ de degradación acelerada. La RT tuvo una disminución de menos de $20 \%$ después de $180 \mathrm{~h}$ de exposición, este valor se mantuvo constante durante el resto del experimento, mostrando la típica resistencia a la degradación de los polímeros sintéticos (Mucha y Pawlak 2005). Por otro lado, la DR sufrió un cambio importante, disminuyendo aproximadamente $80 \%$ a las $180 \mathrm{~h}$, reteniendo sólo $10 \%$ de su deformación original al final del experimento.

El MY de las películas A y B (Fig. 4b y c) mostró una disminución de $10 \%$ después de 180 h de degradación acelerada para después presentar un aumento importante a las $270 \mathrm{~h}$, alcanzando un valor de $110 \%$. Este aumento pudo ser debido a entrecruzamientos que se dan después del rompimiento de cadenas moleculares (Sionkowska et al. 2011) y que pueden producir un aumento en la rigidez del material. Los valores de MY disminuyeron en mayor medida a las $360 \mathrm{~h}$ de exposición. Aparentemente, después de las $270 \mathrm{~h}$ de degradación acelerada comienza el proceso de fotooxidación del quitosano.

Las películas de $\mathrm{PE}$ /quitosano también mostraron un descenso en los valores de RT (Fig. 4b), registrándose una disminución de 25 a $30 \%$ después de 180 h de degradación acelerada. A las 270 h se observó también un aumento ligero en los valores de RT, lo que confirma el aumento en la rigidez producido por la fotodegradación en las mezclas de polímeros sintéticos con quitosano. Después de $360 \mathrm{~h}$, los valores de RT de las películas A y B muestran una pérdida de $40 \%$, esto es un indicio de una aceleración de los procesos de degradación en aquellas que contienen
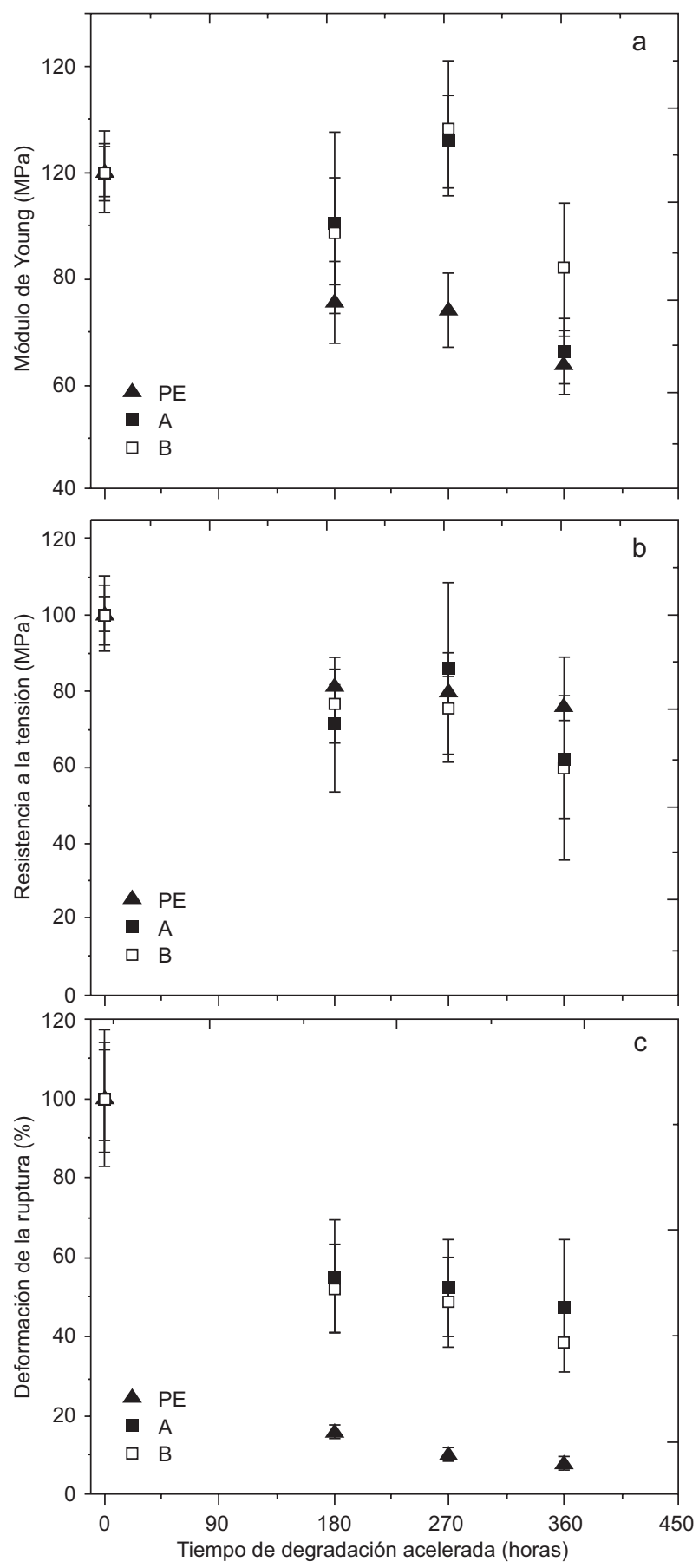

Fig. 4. Retención del (a) módulo de Young, (b) resistencia a la tensión y (c) deformación a la ruptura de las películas de polietileno, A y B, en función del tiempo de exposición acelerada. $\mathrm{Mpa}=$ megapascal

quitosano en comparación con la película de PE, que sólo pierde $20 \%$ del RT. Los valores de RT para las películas compatibilizadas son menores que los de las películas compatibilizadas después de $270 \mathrm{~h}$ de degradación acelerada, este es otro indicativo del 
efecto del PEgMA, que favorece la degradación natural de las películas de PE con quitosano.

Desde las $180 \mathrm{~h}$ de exposición, las películas A y $\mathrm{B}$ se tornaron rígidas, mostrando una retención de su DR de $50 \%$. Se observó una disminución constante de estos valores durante el resto del experimento, este es el comportamiento esperado para los polímeros (Dintcheva et al. 2010).

La adición de quitosano a la matriz de PE afecta significativamente el comportamiento mecánico de las películas (Al-Salem 2009). Los cambios en las propiedades mecánicas de las mismas durante la exposición natural pueden estar asociados con alteraciones fotoquímicas, que producen una disminución del peso molecular debido al rompimiento de cadenas y entrecruzamiento. Por lo tanto, la adición de quitosano a la matriz de PE resulta en un aumento de la degradación de las películas. Lo que está evidenciado por los cambios en las propiedades mecánicas, especialmente la disminución de MY y $\mathrm{RT}$, de las películas que contienen quitosano después de los experimentos de degradación acelerada.

\section{Observación visual}

Al aumentar el tiempo de exposición, las características superficiales de la película de PE cambiaron ligeramente, perdiendo su brillo característico después de $180 \mathrm{~h}$ de exposición. No fue posible observar otro cambio después de ningún periodo de degradación acelerada (Fig. 5a).

Las películas con quitosano y PEgMA (B) mostraron una superficie más homogénea. Las partículas de quitosano fueron menos notorias a simple vista comparadas con aquellas sin compatibilizante (A) (Fig. 5b y c). En los dos casos, el quitosano otorga cierta coloración amarillo claro, en concordancia con lo reportado por otros autores (Butler et al.1996).

Las películas A y B mostraron cambios importantes en apariencia y coloración al estar expuestas en la cámara de degradación acelerada. Inicialmente, la superficie de las películas era suave y casi transparente. A las $180 \mathrm{~h}$ apareció una coloración amarilla que se mantuvo constante durante el resto del experimento para la película A. En el caso de la película B, este color se intensificó en un tono amarillo-café, en función del tiempo de degradación acelerada (Fig. $5 \mathbf{b}$ y c). De acuerdo con Shen et al. (2011), estos cambios de color se relacionan con la aparición de grupos cromóforos como el $\mathrm{C}=\mathrm{O}$ producidos por efecto de la radiación. Esta explicación es acorde con los resultados de los espectros de FT-IR presentados en este artículo $\left(1712 \mathrm{~cm}^{-1}\right)$. Yu et al. (2011) lo explican como un efecto del entrecruzamiento después del rompimiento de

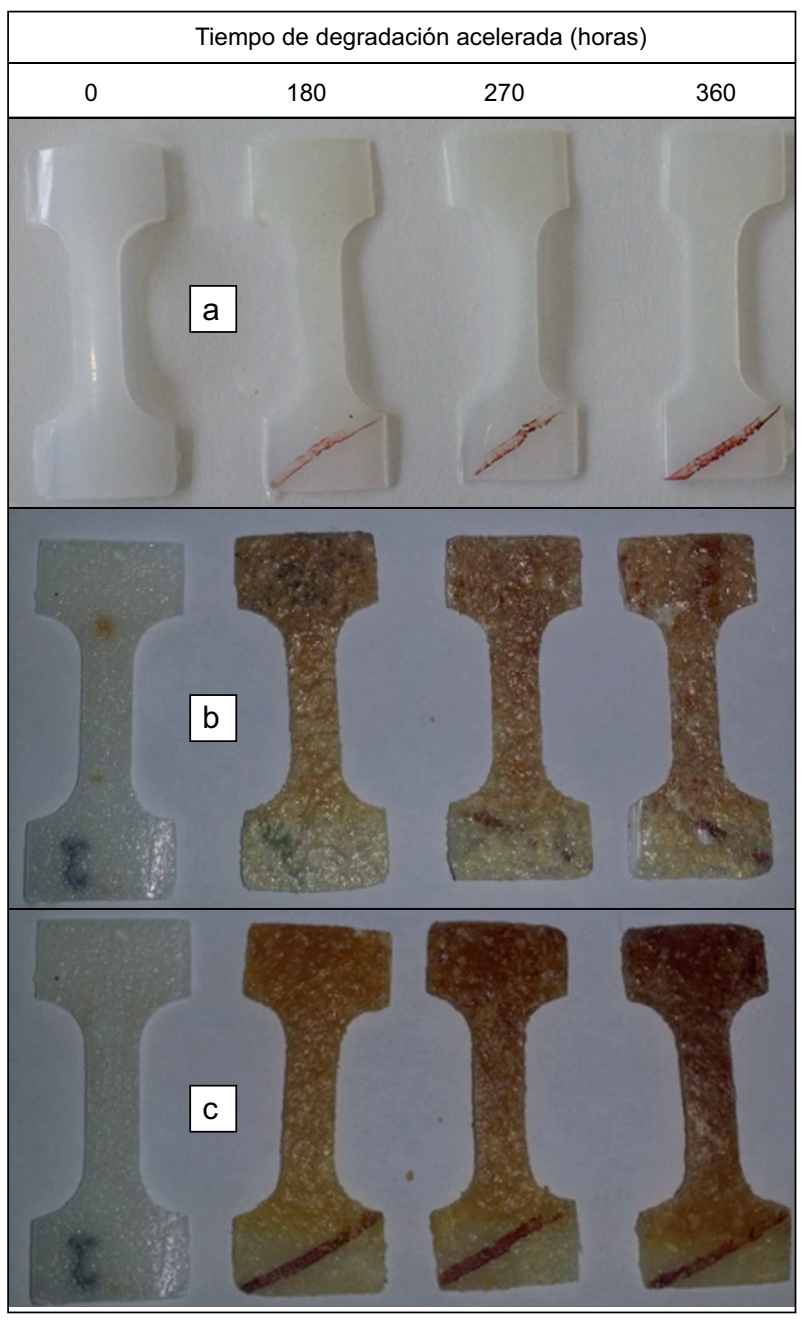

Fig. 5. Imágenes de un espécimen de la película (a) polietileno, (b) Ay (c) B después de distintos periodos de degradación acelerada

cadenas, en donde se forma el grupo $\mathrm{C}=\mathrm{N}$, que también es un cromóforo. Sin embargo, su banda de absorción está en la región UV y no puede ser observada en la región visible. La existencia de otros grupos, como glucosamina o ácidos carboxílicos, pueden actuar como un auxocromo, produciendo un aumento en la longitud de onda de absorción del $\mathrm{C}=\mathrm{N}$-. Para poder ser observada en el rango visible, el quitosano entrecruzado se tornaría de un color oscuro.

El uso de PEgMA como compatibilizante aumentó ligeramente la degradación del material, considerando que la superficie de estas películas presentó una coloración más intensa al término del experimento.

Los resultados del estudio morfológico concuerdan con la medición de propiedades mecánicas, donde las películas de PE puro mostraron una mayor resistencia a la degradación acelerada comparadas 
con las que contienen quitosano. La adición de quitosano a la matriz de PE, aceleró de manera importante el comportamiento de degradación de las películas.

\section{Estudios de degradación en tierra}

La figura 6 muestra la pérdida de peso de las películas en función del tiempo de exposición en tierra de vivero. La película de PE registró una pérdida de peso de $0.05 \%$. El PE presenta una alta resistencia a la degradación en tierra en un ambiente húmedo. La adición de $15 \%$ de quitosano aceleró significativamente la pérdida de peso del material: $10 \%$ en 45 días, $12 \%$ a los 135 días y una perdida máxima de $13 \%$ a los 180 días de exposición en tierra. La incorporación de PEgMA no presentó un efecto importante en la degradación en tierra de las mezclas de PE con quitosano (Fig. 6).

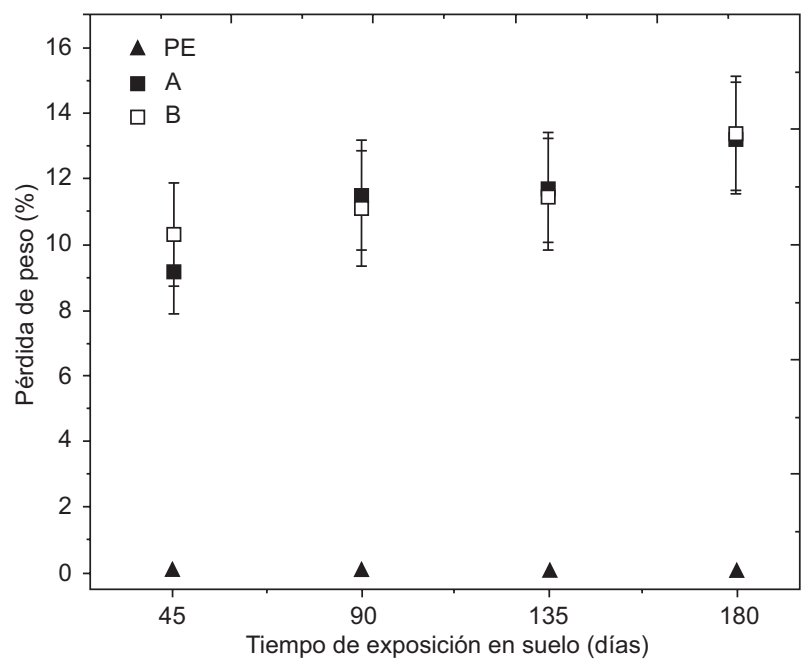

Fig. 6. Gráfica de pérdida de peso en función del tiempo de exposición en tierra de las películas de polietileno, A y B

\section{CONCLUSIONES}

La fotooxidación y la presencia de humedad a alta temperatura provocaron la degradación de los materiales. Una profunda caracterización de las películas, antes y después de la degradación acelerada, reveló un efecto importante del quitosano sobre la degradación de las mismas.

Las películas con $15 \%$ de quitosano perdieron 40 $\%$ del valor inicial de resistencia a la tensión después de 360 h de degradación acelerada. La degradación oxidativa produjo un aumento en el contenido de grupos carbonilo. Esta exposición también generó un aumento en la rigidez del material, con los cambios asociados en sus propiedades mecánicas. La presencia de PEgMA como compatibilizante produce un ligero aumento en la degradación de las películas de $\mathrm{PE}$ con quitosano, comparadas con las películas sin compatibilizar.

Las condiciones a las que estuvieron expuestas las películas, en la cámara de degradación QUV, favorecieron una rápida degradación del quitosano. Por otra parte, las películas con quitosano presentaron una mayor pérdida de peso al estar enterradas en tierra de vivero en un ambiente húmedo en comparación con las de PE. Los datos relacionados a la degradación de las mezclas de PE y quitosano reportados en este trabajo son importantes en el estudio del quitosano como una solución favorable con el ambiente ante el problema de la disposición de plásticos desechables.

\section{REFERENCIAS}

Al-Salem S.M. (2009). Influence of natural and accelerated weathering on various formulations of linear low density polyethylene (LLDPE) films. Mater and Design 30 (5), 1729-1736. DOI: 10.1016/j.matdes.2008.07.049

Andrady A. (1997). Wavelength sensitivity in polymer photodegradation. Adv. Polym. Sci. 128 (1), 49-94. DOI: $10.1007 / 3-540-61218-16$

Andrady A.L., Hamid S.H., Hu X. y Torikai A. (1998). Effects of increased solar ultraviolet radiation on materials. J. Photoch. Photobio B. 46 (1-3), 96-103. DOI: $10.1016 / \mathrm{S} 1011-1344(98) 00188-2$

Butler B.L., Vergano V.J., Testin R.F., Bunn J.M. y Wiles J.L. (1996). Mechanical and barrier properties of edible chitosan films as affected by composition and storage. J. Food Sci. 5 (5), 953-956. DOI: 10.1111/j.1365-2621.1996.tb10909.x

Chuensangjun C., Pechyen C. y Sirisansaneeyakul S. (2013). Degradation behaviors of different blends of polylactic acid buried in soil. Energy Procedia 34 (1), 73-82.

DOI: 10.1016/j.egypro.2013.06.735

Corrales T., Catalina F., Peinado C., Allen N. y Fontan E. (2002). Photooxidative and thermal degradation of polyethylenes: interrelationship by chemiluminescence, thermal gravimetric analysis and FTIR data. J. of Photoch. Photobio. 147 (3), 213-224. DOI: 10.1016/S1010-6030(01)00629-3

Day M., Shaw K., Cooney D., Watts J. y Harrigan B. (1997). Degradable polymers: The role of the degradation environment. J. Environ. Polym. Degr. 5 (3), 137-151. DOI: 10.1007/BF02763657

Del Castillo-Castro T., Castillo-Ortega M.M., HerreraFranco P.J. y Rodríguez-Félix D.E. (2011). Compatibilization of polyethylene/polyaniline blends with 
polyethylene-graft-maleic anhydride. J. Appl. Polym. Sci. 119 (5), 2895-2901. DOI: 10.1002/app.32971

Dintcheva N.T., Al-Malaika S. y La Mantia F.P. (2009). Effect of extrusion and photo-oxidation on polyethylene/clay nanocomposites. Polym. Degrad. Stabil. 94 (9), 1571-1588.

DOI: 10.1016/j.polymdegradstab.2009.04.012

Dintcheva N.T., Filippone G., La Mantia F.P. y Acierno D. (2010). Photo-oxidation behaviour of polyethylene/ polyamide 6 blends filled with organomodified clay: Improvement of the photo-resistance through morphology modification. Polym. Degrad. Stabil. 95 (4), 527535. DOI: 10.1016/j.polymdegradstab.2009.12.021

Dostál J., Kasparkova V., Zatloukal M., Muras J. y Simek L. (2008). Influence of the repeated extrusion on the degradation of polyethylene. Structural changes in low density polyethylene. Eur. Polym. J. 44 (8), 2652-2658. DOI: 10.1016/j.eurpolymj.2008.05.028

Ermolovich O.A. y Makarevich A.V. (2006). Effect of compatibilizer additives on the technological and performance characteristics of biodegradable materials based on starch-filled polyethylene. Russ. J. Appl. Chem. 79 (9), 1526-1531. DOI: $10.1134 / \mathrm{S} 1070427206090266$

Freile-Pelegrín Y., Madera-Santana T., Robledo D., Veleva L., Quintana P. y Azamar J.A. (2007). Degradation of agar films in a humid tropical climate: Thermal, mechanical, morphological and structural changes. Polym. Degrad. Stab. 92 (2), 244-252.

DOI: 10.1016/j.polymdegradstab.2006.11.005

Guadagno L., Naddeo C., Vittoria V., Camino G. y Cagnani C. (2001). Chemical and morphological modifications of irradiated linear low density polyethylene (LLDPE). Polym. Degrad. Stab. 72 (1), 175-186.

DOI: 10.1016/S0141-3910(01)00024-6

Gugumus F. (1993). Re-evaluation of the stabilization mechanisms of various light stabilizer classes. Polym. Degrad. and Stab. 39 (1), 117-135.

DOI: 10.1016/0141-3910(93)90131-2

Harish-Prashanth K.V. y Tharanathan R.N. (2007). Chitin/ chitosan: modifications and their unlimited application potential, an overview. Trends Food Sci. Tech. 18 (3), 117-131. DOI: 10.1016/j.tifs.2006.10.022

Mathew S. y Abraham T.E. (2008). Characterisation of ferulic acid incorporated starch-chitosan blend films. Food Hydrocolloid. 22 (5), 826-835.

DOI: 10.1016/j.foodhyd.2007.03.012

Mucha M. y Pawlak A. (2005). Thermal analysis of chitosan and its blends. Thermochim Acta. 427(1-2), 69-76. DOI: $10.1016 /$ j.tca.2004.08.014
Nair L.S. y Laurencin C.T. (2007). Biodegradable polymers as biomaterials. Progress in Polymer Science 32 (8-9), 762-798. DOI: 10.1016/j.progpolymsci.2007.05.017

Ojeda T., Freitas A., Birck K., Dalmolin E. y Jacques R. (2011). Degradability of linear polyolefins under natural weathering. Polym. Degrad. Stab. 96 (4), 703-707. DOI: 10.1016/j.polymdegradstab.2010.12.004

Pillai C.K.S., Paul W. y Sharma C.P. (2009). Chitin and chitosan polymers: Chemistry, solubility and fiber formation. Prog. Polym. Sci. 34 (7), 641-678. DOI: 10.1016/j.progpolymsci.2009.04.001

Quiroz-Castillo J.M., Rodríguez-Félix D.E., GrijalvaMonteverde H., Del Castillo-Castro T., PlascenciaJatomea M., Rodríguez-Félix F. y Herrera-Franco P.J. (2014). Preparation of extruded polyethylene/chitosan blends compatibilizedwith polyethylene-graft-maleic anhydride. Carbohyd. Polym. 101 (30), 1094-1100. DOI: 10.1016/j.carbpol.2013.10.052

Rogovinaa S.Z., Aleksanyana K.V., Novikova D.D., Pruta E.V. y Rebrovb A.V. (2009). Synthesis and investigation of polyethylene blends with natural polysaccharides and their derivatives. Polym. Sci. Ser. A. 51 (5), 554-562. DOI: 10.1134/S0965545X09050101

Shen K., Hu Q., Wang Z. y Qu J. (2011). Effect of ${ }^{60} \mathrm{Co}$ irradiation on the properties of chitosan rod. Mater. Sci. Eng. C. 31 (5), 866-872.

DOI: 10.1016/j.msec.2011.02.002

Singh G., Bhunia H., Rajor A. y Choudhary V. (2011). Thermal properties and degradation characteristics of polylactide, linear low density polyethylene, and their Blends. Polym. Bull. 66 (7), 939-953.

DOI: $10.1007 / \mathrm{s} 00289-010-0367-\mathrm{x}$

Singh G., Bhunia H., Rajor A. y Choudhary V. (2011). Thermal properties and degradation characteristics of polylactide, linear low density polyethylene, and their Blends. Polym. Bull. 66 (7), 939-953.

DOI: $10.1007 / \mathrm{s} 00289-010-0367-x$

Yu Q., Song Y., Shi X., Xu C. y Bin Y. (2011). Preparation and properties of chitosan derivative/poly(vinyl alcohol) blend film crosslinked with glutaraldehyde. Carbohyd. Polym. 84 (1), 465-470.

DOI: $10.1016 /$ j.carbpol.2010.12.006

Zhong C., Wu J., Reinhart-King C.A. y Chu C.C. (2010). Synthesis, characterization and cytotoxicity of photocrosslinked maleic chitosan-polyethylene glycol diacrylate hybrid hydrogels. Acta Biomater. 6 (10), 3908-3918. DOI: 10.1016/j.actbio.2010.04.011 